\title{
Hepatitis with Fibrin-Ring Granulomas
}

\author{
B. Khanlari, M. Bodmer, L. Terracciano, M.H. Heim, U. Fluckiger, M. Weisser
}

\begin{abstract}
We describe a 66-year-old woman hospitalized with fever, fatigue and hepatopathy. In her medical history arterial hypertension (treated with propranolol and lisinopril), diabetes mellitus type 2 (no treatment before admission) and a gout arthropathy were noted wherefore a therapy with allopurinol $300 \mathrm{mg}$ per day has been started 4 months before. Liver biopsy revealed fibrin-ring granulomas, compatible with allopurinolinduced hepatitis. Because of persistence of high fever after stopping allopurinol, steroids ( $1 \mathrm{mg} / \mathrm{kg}$ ) were started. Under this treatment, she developed pancytopenia and fever. The bone marrow aspiration revealed Leishmania infantum. A second liver biopsy showed amastigotes and a disappearance of the granulomas. The history revealed a travel to Malta 2 years earlier. Despite adequate treatment with liposomal amphotericin $B$ the patient deteriorated and finally died in septic shock.
\end{abstract}

Infection 2008; 36: 381-383

DOI 10.1007/s15010-007-6365-8

\section{Introduction}

Fibrin-ring granulomas in liver biopsy specimens of acute hepatitis are a rare and unspecific finding. Possible causes include Morbus Hodgkin, drug reactions, rheumatic diseases and infections. Pathogens associated with fibrin-ring granulomas are Coxiella burnetii, Mycobacterium tuberculosis and Leishmania spp. Leishmaniasis can be reactivated in immunocompromised patients years after primary infection. Typical clinical symptoms may then be absent, leading to a delay in diagnosis and more severe course of infection. Due to human migration and increased tourism, visceral leishmaniasis may become more common in non-endemic areas.

\section{Case}

A 66-year-old woman was referred for progressive weakness, fatigue, fever and dizziness over the last 3 weeks. In the medical history arterial hypertension, diabetes mellitus type 2 and a gout arthropathy were noted. Medication consisted of lisinopril $5 \mathrm{mg}$, propranolol $40 \mathrm{mg}$ and allopurinol $300 \mathrm{mg}$ daily started 4 months ago.

At admission the patient was in a reduced condition and febrile $\left(38^{\circ} \mathrm{C}\right)$. The physical examination revealed adipositas $\left(\mathrm{BMI} 31 \mathrm{~kg} / \mathrm{m}^{2}\right)$, edema of the lower extremities and spleno- megaly. Blood tests showed elevated liver enzymes (ASAT $202 \mathrm{U} / 1$ [11-36 U/1], ALAT $89 \mathrm{U} / 1$ [10-37 U/1], bilirubin $19.7 \mu \mathrm{mol} / \mathrm{l}[5-18 \mu \mathrm{mol} / \mathrm{l}], \mathrm{gGT} 243 \mathrm{U} / \mathrm{l}$ [8-49 U/1] and alkaline phosphatase $236 \mathrm{U} / 1$ [31-108 U/1]). The platelet count was low $\left(72 \times 10^{9} / 1\right)$, the absolute number of leukocytes was normal with shift to band forms (25\%). An abdominal ultrasound showed hyperechogenity of the liver and a splenomegaly of $14 \times 6 \mathrm{~cm}$. Serologic tests for HBV, HAV and HCV were negative.

A liver biopsy revealed an extensive granulomatous hepatitis with fibrin-ring granulomas (Figure 1a, b). Possible etiologies of fibrin-ring granulomas as infections with Coxiella burnetii, Rickettsia spp., Leptospira spp., Salmonella spp., CMV and HIV were excluded by serologies and/or cultures. M. tuberculosis was not found in the liver biopsy (Ziehl-Neelsen, PCR and cultures). Antimitochondrial antibodies, antineutrophil cytoplasmic antibodies and antinuclear antibodies were negative. A bone marrow biopsy and a computer tomography did not show signs of lymphoma. Neither in the biopsy of the liver nor that of the bone marrow were amastigotes detected. Finally, the granulomatous hepatitis was interpreted as allopurinol-associated and allopurinol was stopped. Because of the persisting high fever and discomfort of the patient a treatment with prednisone $75 \mathrm{mg}$ per day was started and the dosage was slowly tapered. The patient's condition improved and she was discharged.

After 3 weeks of therapy with a total of $1,050 \mathrm{mg}$ prednisone, the patient was readmitted with pancytopenia (platelets $20 \times 10^{9}$ per liter, hemoglobin $110 \mathrm{~g} / \mathrm{l}$, leukocytes $0.97 \times 10^{9} \mathrm{per}$ liter, neutrophils $0.56 \times 10^{9}$ per liter) and persistently elevated liver enzymes (ASAT $104 \mathrm{U} / 1$, ALAT $91 \mathrm{U} / 1$, gGT 1,188 U/l, alkaline phosphatase $305 \mathrm{U} / \mathrm{l}$, bilirubin $68 \mu \mathrm{mol} / \mathrm{l})$. The liver and bone marrow biopsies were repeated. Now an extensive infiltration with amastigotes was seen in the liver (Figure 2) and bone marrow (Figure 3), identified as Leishmania infantum by

\footnotetext{
B. Khanlari

Dept. Internal Medicine, University Hospital, Basel, Switzerland M. Bodmer

Division of Clinical Pharmacology and Toxicology, University Hospital, Basel, Switzerland

L. Terracciano

Division of Pathology, University Hospital Basel, Basel, Switzerland

M.H. Heim

Division of Gastroenterology, University Hospital, Basel, Switzerland

U. Fluckiger, $\mathbf{M}$. Weisser (corresponding author)

Division of Infectious Diseases and Hospital Epidemiology, Dept. of Internal Medicine, University Hospital Basel, Petersgraben 4, 4031 Basel, Switzerland; Phone: (+41/61) 328 6550, Fax: 265 3198,

e-mail: weisserm@uhbs.ch
}

Received: December 19, 2006 - Revision accepted: April 24, 2007

Published online: October 9, 2007 
Figure 1. (a) Liver parenchyma showing preserved lobular architecture, macrovesicular steatosis and diffuse granulomatous hepatitis with several fibrin-ring granulomas (H.E. ×100). (b) The granulomas display a central fatty vacuole and the surrounding macrophages are trapped in a mesh of fibrin (H.E. $\times 400)$.
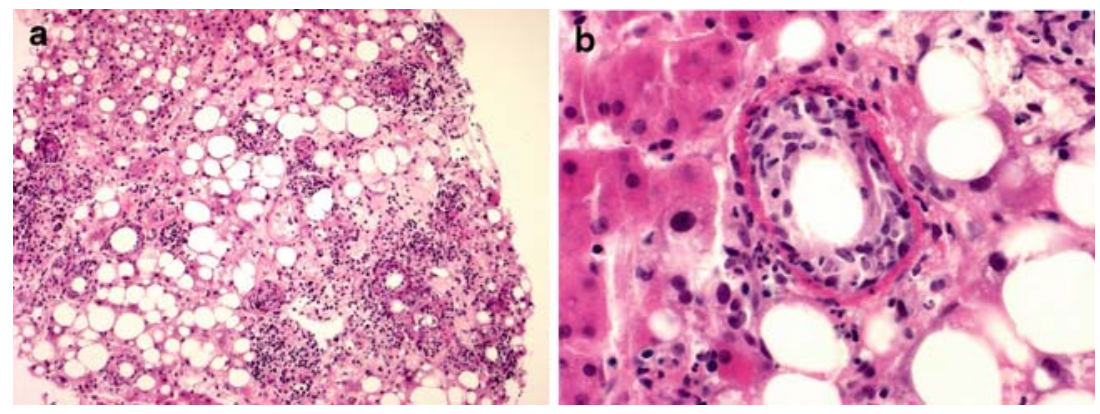

polymerase chain reaction (restriction fragment length polymorphism, PCR-RFLP [1]) in blood and liver biopsy. Also the serology (immunofluorescence test) for leishmania infantum was strongly positive. There was no evidence of fibrinous granulomas anymore in the liver biopsy. The travel history revealed a vacation in the Mediterranean island of Malta 2 years before. A therapy with liposomal amphotericin B $3 \mathrm{mg} / \mathrm{kg}$ was started and well tolerated. After 8 days of therapy the patient developed pneumonia with Pneumocystis jiroveci and multiorgan failure and she died 23 days after initiation of antiparasitic treatment. The autopsy showed only a discrete persistent infiltration of amastigotes in the liver and bone marrow. Retrospectively the initial bone marrow and liver biopsies were reexamined by an experienced pathologist. No amastigotes were found in those specimens. The PCR analysis was not repeated in the biopsies.

\section{Discussion and Conclusion}

The differential diagnosis of granulomatous hepatitis is broad. Sarcoidosis, tuberculosis, primary biliary cirrhosis and drug-induced hepatitis are the most common etiologies. Fibrin-ring granulomas are rare and can be due to infections with C. burnetii, EBV, CMV, Hepatitis A or Leishmania spp., Morbus Hodgkin and drug toxicity from allopurinol [2]. In our case infectious etiologies were not found by serology and cultures, the liver and bone marrow biopsies were negative for amastigotes and there was no evidence of lymphoma in the abdominal CT scan.

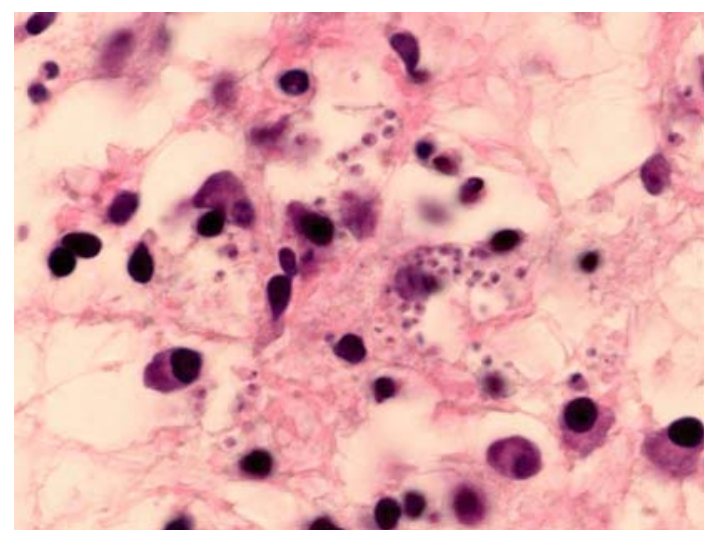

Figure 2. Several parasites are present in Kupffer cells and in some hepatocytes (amastigote form of Leishmania) (H.E. $\times 400$ ).
After exclusion of infectious and malignant causes, the diagnosis of allopurinol-induced hepatitis was made, although the clinical presentation with edema and splenomegaly was not typical and features of an allergic drug reaction like rash, arthralgias and eosinophilia were lacking.

In the allopurinol-associated hypersensitivity syndrome, clinical symptoms are evident most often 4-6 weeks after initiation of therapy, including fever, rash, arthralgias and eosinophilia. Usually the patients recover within 6 weeks after cessation of allopurinol [3]. Our patient was hospitalized because of fever and hepatopathy 4 months after starting allopurinol.

Besides heterogeneous clinical manifestations, Leishmania infections can remain asymptomatic, especially in immunocompetent patients [4], whereas, severe disease is possible despite low parasite load. The liver involvement in visceral leishmaniasis may appear as chronic granulomatous hepatitis [5]. Fulminant hepatitis has been described in 3 of 150 immunocompetent children with Kala-azar in an Indian study [6].

Visceral leishmaniasis is usually diagnosed in bone marrow, spleen, lymph nodes and liver biopsy [7-9]. The bone marrow and liver biopsies in our patient were initially negative for Leishmania. Retrospectively, it is not

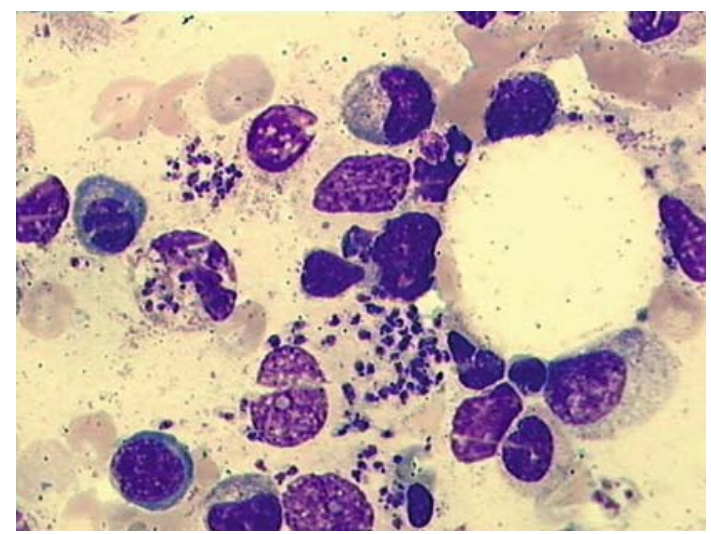

Figure 3. Bone marrow biopsy showing numerous Leishmania (Giemsa, ×630). 
clear whether the initial finding of fibrin-ring granulomas was due to occult visceral leishmaniasis or to allopurinol therapy. Interestingly, allopurinol is used in combination with antimoniate for the treatment of cutaneous leishmaniasis [10], while in visceral leishmaniasis the results of this combination therapy are not so promising, either because of relapses [11] or of lacking effectiveness [12]. In combination with ketoconazole, allopurinol was reported to be successful for visceral leishmaniasis in renal transplantation although in a higher dose than that used in the treatment of gout arthropathy (600 mg vs. $300 \mathrm{mg}$ ) [13]. One could hypothesize therefore, that allopurinol led to a disappearance of the parasites, while the granulomatous hepatitis persisted. Furthermore, in the natural course of leishmaniasis, formation of granulomas is a sign of strong cellular immune response with few parasites found in examined tissue and an a- to oligo-symptomatic state of disease.

After stopping the treatment and starting an immunosuppressive therapy with prednisone, a progression to fulminant leishmaniasis occurred, and the liver granulomas disappeared. Steroids, alone or in combination with other immunosuppressive medications, are a known risk factor for severe courses of infection with Leishmania $[14,15]$.

Our patient must have acquired the infection two years before the start of symptoms on a vacation in Malta, where L. infantum is endemic. Prolonged latent infection with the appearance of symptoms only after immunosuppression was started has been described [16, 17].

In conclusion, like other emerging infectious diseases visceral leishmaniasis becomes more common in non-endemic regions with migration and tourism. Therefore, a travel history is mandatory in all patients. In patients with alterated immune status (e.g., disease or therapy-associated) and granulomatous hepatitis, leishmaniasis has to be strongly considered and searched repeatedly. In patients with fever, hepatosplenomegaly and pancytopenia with a travel history to endemic areas, serology for Leishmania spp. is a useful screening test.

\section{References}

1. Marfurt J, Niederwieser I, Ntoh DM, Beck HP, Felger I: Diagnostic genotyping of old and new world leishmania species by PCRRFLP. Diagn Microbiol Infect Dis 2003; 46: 115-124.
2. Marazuela M, Moreno A, Yebra M, Cerezo M, Gomez-Gesto M, Vargas JA: Hepatic fibrin-ring granulomas: a clinicopathologic study of 23 patients. Hum Pathol 1991; 22: 607-613.

3. Stricker BH, Blok AP, Babany G, Benhamou JP: Fibrin ring granulomas and allopurinol. Gastroenterology 1989; 96: 1199-1203.

4. le Fichoux Y, Quaranta JF, Aufeuvre JP, Lelievre A, Marty P, Suffia I, Rousseau D, Kubar J: Occurrence of Leishmania infantum parasitemia in asymptomatic blood donors living in an area of endemicity in southern France. J Clin Microbiol 1999; 37: 1953-1957.

5. el Hag IA, Hashim FA, el Toum IA, Homeida M, el Kalifa M, el Hassan AM: Liver morphology and function in visceral leishmaniasis (Kala-azar). J Clin Pathol 1994; 47: 547-551.

6. Singh UK, Sinha RK, Sharma VK: Fulminant hepatitis in Kalaazar. Indian J Pediatr 1995; 62: 571-574.

7. Kumar PV, Omrani GH, Saberfirouzi M, et al. Kala-azar: liver fine needle aspiration findings in 23 cases presenting with a fever of unknown origin. Acta Cytol 1996; 40: 263-268.

8. Artan R, Yilmaz A, Akçam M, Aksoy NH: Liver biopsy in the diagnosis of visceral leishmaniasis. J Gastroenterol Hepatol 2006; 21: 299-302.

9. Guerin PJ, Olliaro P, Sundar S, Boelaert M, Croft SL, Desjeux P, Wasunna MK, Bryceson AD: Visceral leishmaniasis: current status of control, diagnosis, and treatment, and a proposed research and development agenda. Lancet Infect Dis 2002; 2: 494-501.

10. Iraj Esfandiarpour MD, Afsáneh Alavi MD: Evaluating the efficacy of allopurinol and meglumine antimoniate (Glucantime) in the treatment of cutaneous leishmaniasis. Int J Dermatol 2002; 41: 521-524.

11. Berman JD: Chemotherapy for leishmaniasis: biochemical mechanisms, clinical efficacy, and future strategies (review). Rev Infect Dis 1988; 10: 560-586.

12. Singh NK, Jha TK, Singh IJ, Jha S: Combination therapy in Kalaazar. J Assoc Physicians India 1995; 43: 319-320.

13. Llorente S, Gimeno L, Navarro MJ, Moreno S, Rodriguez M: Therapy of visceral leishmaniasis in renal transplant recipients intolerant to pentavalent antimonials. Transplantation 2000; 70: 800-801.

14. Garcia-Cordoba F, et al. Fatal visceral leishmaniasis, with massive bone-marrow infection, in an immunosuppressed but HIVnegative Spanish patient, after the initiation of treatment with meglumine antimoniate. Ann Trop Med Parasitol 2005; 99: 125-130.

15. Scatena P: Visceral leishmaniasis in a patient treated for polyarteritis nodosa. Clin Exp Rheumatol 2003; 21: 121-123.

16. Malik A: Changing pattern of visceral Leishmaniasis, United Kingdom, 1985-2004. EID, 12: 1257-1259.

17. Badaro R: Leishmania donovani: an opportunistic microbe associated with progresssive disease in three immunocompromised patients. Lancet 1986; 647-649. 